\title{
Long-term disease control and high clinical benefit in a patient with advanced thyroid cancer treated with lenvatinib
}

\author{
Leonardo Muratori*,1, Paola Sperone ${ }^{1}$ \& Giorgio Vittorio Scagliotti ${ }^{1}$ \\ ${ }^{1}$ Medical Oncology, Department of Oncology, University of Turin, Azienda Ospedaliera Universitaria San Luigi Gonzaga, Regione \\ Gonzole 10, Orbassano 10043, Turin, Italy \\ *Author for correspondence: Tel.: +39393 7010166; muratorileonardo@gmail.com
}

We report a case of a 37-year-old man with metastatic differentiated thyroid carcinoma, previously submitted to total thyroidectomy, radio-iodine therapy and lung metastasectomy, who underwent systemic treatment with lenvatinib for tumor recurrence in the lung, mediastinal lymph nodes, left gluteus and left orbit. Lenvatinib induced rapid and durable disease regression; the drug effect has continued after $>1$ year, as well as a very considerable clinical benefit. The results achieved by lenvatinib in treatment of metastatic differentiated thyroid carcinoma are clear and irrefutable. Real-life data, obtained by case reports and retrospective studies, are equally important to increase the knowledge about this drug and improve the clinical management.

First draft submitted: 19 February 2019; Accepted for publication: 18 July 2019; Published online: 6 August 2019

Keywords: case report $\bullet$ lenvatinib • thyroid cancer

Differentiated thyroid carcinoma (DTC) is the most common malignant tumor of the thyroid gland. Although the incidence of this cancer has been increasing over the last few decades, mortality is constantly decreasing, owing mainly to improvements in early diagnosis and local treatment [1]. In fact, low-stage disease, which is amenable to surgical resection, has excellent prognosis [2]. Post-surgical radioiodine $\left({ }^{131} \mathrm{I}\right)$ therapy is not generally indicated in T1 and T2 stages even though it could be considered in case of presence of other risk factors (more aggressive histological types, positive lymph nodes, history of exposure to radiation) [3]. 131I therapy is generally recommended for adjuvant treatment for T3 and T4 patients, with the aim of reducing the risk of disease relapse [4]. Distant metastatic spread occurs in a minority of cases (approximately 10\%), with lung and bones being the most commonly involved sites. Thyroid cancer that relapses during or after adjuvant 131I therapy carries a poor prognosis because of refractoriness to conventional anticancer treatments. Historically, surgical resection of metastases and external beam radiotherapy, when feasible, has represented suitable treatment options. Increased knowledge in the molecular biology of thyroid cancer has clarified the complex signaling networks that sustain this cancer phenotype. These involve angiogenesis, BRAF, NRAS, HRAS, RET/DTC, FGFR and PDGFR signaling. As a consequence, a strong rationale supported the clinical evaluation of multitargeted tyrosine kinase inhibitors (TKIs). One of these agents, sorafenib, was investigated in a randomized Phase III trial that included 417 patients, where it resulted superior to placebo in terms of progression-free survival (PFS; 10.8 vs 5.4 months) [5]. More recently, lenvatinib, an oral multitargeted TKI that inhibits VEGFR1-3, PDGFR- $\alpha$, FGFR1-4, RET and KIT - all targets implicated in signaling networks that promote angiogenesis [6] - was approved on the basis of a Phase III trial enrolling 360 patients with iodine-refractory thyroid cancer, who were randomized to lenvatinib $24 \mathrm{mg} /$ day or placebo. PFS was significantly higher in lenvatinib group (median PFS: 18.2 vs 3.6 months, hazard ratio: 0.21; 99\% CI: 0.14-0.31; $\mathrm{p}<0.001$ ), although no advantage in overall survival was observed [7]. Based on these results, lenvatinib was approved for the treatment of patients with radioiodine-refractory metastatic DTC (MDTC). Here, we report the case of a young patient with MDTC treated with lenvatinib after total thyroidectomy, ${ }^{131} \mathrm{I}$ therapy and lung metastasectomy. The patient achieved a long-term response on all sites of metastasis and a very important clinical 
benefit with complete resolution of all symptoms. The collection of clinical cases concerning this uncommon neoplasm could help the clinicians in the management of daily practice.

\section{Presentation of case}

A 37-year-old man underwent total thyroidectomy in October 2013 because of the incidental finding of a thyroid lesion. Histological examination showed a papillary carcinoma, staged $\mathrm{T} 3 \mathrm{Nx}$, according to the seventh edition of Tumor, Node, Metastasis staging. No distant metastases were detected by total body CT scan. Because of the high stage, the patients received an adjuvant therapy with $131 \mathrm{I}(0.7 \mu \mathrm{g} / \mathrm{kg} /$ day in 3 days $)$. The post ${ }^{131} \mathrm{I}$ CT-scan showed no residual disease and the follow-up was unremarkable until September 2015 when a routine blood test revealed an increase in the levels of high-sensitivity human thyroglobulin. The 18-fluorodeoxyglucose (18-FDG) PET CT scan showed significant radiotracer uptake in the right lung. In November 2015, the patient underwent resection of the lung lesions, with histopathological examination confirming metastatic thyroid cancer, with oxyphil cells and low differentiated features. Based on no other clear evidence of distant metastases after surgical resection, the patient resumed regular clinical follow-up. In May 2016, multiple bilateral lung lesions were detected by a routine total body CT scan. Considering the small size of the new nodules and the absence of symptoms, the treating physician opted for a wait-and-see approach. Repeat CT scans revealed no signs of progression until March 2017, when, in spite of stable numerical and dimensional disease in the lung, a new 16-mm nodule was detected in the right kidney. Concomitantly, the patient presented with pain in left gluteus muscle, diplopia and vision impairment of the left eye. A CT scan of the head showed thickening of medial rectus muscle, with deviation and compression of homolateral optical nerve. An 18-FDG PET-CT scan revealed pathological uptake in left orbit, in the mediastinal lymph nodes and in the left gluteus, confirming the clinical suspicion of disease progression. A multidisciplinary evaluation was performed, and it was decided to treat the patient with radiotherapy to the right retrobulbar space. This consisted of 44 Gy administered in 22 fractions, from June to July 2017, with a partial benefit on visual symptoms. In August 2017, systemic therapy with lenvatinib was started. The patient received the recommended dose of $24 \mathrm{mg}$ daily and underwent clinical and laboratory evaluation initially every 2 weeks for 2 months, and subsequently every month. Arterial blood pressure, physical examination, serum chemistry and hematology were evaluated at each visit. Serum thyroid hormone concentration, ECG and echocardiogram were repeated every 3 months. The first CT scan performed in October 2017, showed partial response of disease in all sites of metastases. However, the full dose of lenvatinib was not well tolerated, in particular, in terms of recurrent grade 3 hypertension, according to the common terminology criteria for adverse events version 4.03. According to the insert package, the drug was reduced to $20 \mathrm{mg}$ daily with no subsequent recurrence of clinically relevant hypertension. Cardiac and thyroid functions remained within in the normal range. Patient symptoms, including visual disturbances that had remained after radiotherapy, resolved completely within the first 6 months of treatment with lenvatinib. The 3-monthly follow-up CT scans showed no signs of disease progression. The following CT scans, performed every 3 months, showed no signs of disease progression, and even a progressive reduction of all sites of metastases. The last CT scan, performed in early May 2019, confirmed this trend, showing an additional response of all sites of metastases. At more than 22 months from the start of systemic therapy, the patient is asymptomatic and with no major treatment-related side effects.

\section{Discussion \& conclusion}

Multitargeted TKIs represent a relevant therapeutic option for patients with iodine-resistant metastatic DTC, a condition that had very limited therapeutic options before the advent of these drugs [5-7]. By targeting multiple pathways that underlie this cancer phenotype, lenvatinib has confirmed a remarkable effect on PFS, compared with placebo, in a large randomized clinical trial [7]. Off-target activity, which represents the downside of these drugs, has resulted in a spectrum of side effects that should account for when deciding when and how to use this drug in the clinical practice. Indeed, based on the results of the pivotal trial, about six out of ten patients are expected to show disease response and median duration of response is 30 months, which translates in very long drug exposure in the majority of treated patients [8]. The case that we discuss here is interesting for a number of practical reasons. First, the drug induced rapid disease regression, as revealed by improvement in related disease symptoms (gluteal pain) and radiological evidence. One of the first aims of the treatment of metastatic disease is symptom palliation and, in this case, further improvement occurred in the initial months of treatment, leading to complete and long-lasting resolution of patient initial complaints. Second, the patient has been on treatment for more than 1 year now and, except for the initial 2 months, he now undergoes monthly visits to check tolerance 
and toxicity. Indeed, management of toxicities is an important issue, treatment is to be continued until disease progression and the quality of life is an essential end point to consider. Multitargeted TKIs are generally associated with several adverse effects, including hypertension, oral mucositis, fatigue, increased risk of thromboembolism, hepatotoxicity, skin changes, nausea, diarrhea, anorexia and thyroid function alteration [9]. For this reason, an adequate follow-up of these patients in order to introduce the opportune countermeasures when necessary is fundamental. These potential side effects may negatively impact the quality of life, resulting frequently in dose reduction or treatment discontinuation. In the case, we described here, only a dose reduction from 24 to $20 \mathrm{mg} / \mathrm{day}$ was necessary when grade 3 hypertension occurred, with no further recurrence of this side effect. The clinical benefit was particularly interesting, especially concerning the ocular symptoms. The patient developed a metastasis in the orbital cavity, which affected his sight, producing diplopia and reduction of visual acuity. As described above, this lesion was treated with radiotherapy according to standard of care. Residual sight impairment resolved completely during systemic therapy with lenvatinib. Concomitantly, pain reported at the start of lenvatinib therapy and that required analgesic disappeared after approximately 1 month of treatment, with consequent withdrawal of pain medications. One final point emerges from this clinical case, which - due to the efficacy of lenvatinib - should not be considered 'anecdotal' but yet a frequent occurrence. Our patient underwent surgical treatment of the first metastatic occurrence and local therapy to a critical orbital lesion. These two options, although not devoid of potential complications, have always been considered the standard of care in the absence of active systemic drugs. Nowadays, with the availability of drugs yielding high response rate and long-lasting disease control, the question is - what is the role of local therapy in the management of metastatic DTC? In our opinion, it could be reasonable to treat this disease upfront with lenvatinib or with similar drugs that will show comparable or increased efficacy in the future, reserving surgery or radiation therapy either for resistant disease or for particular situations (medullary compression and oligometastatic progression). However, it should be noted that even this approach, although it could be reducing the local treatment related comorbidities, is not without limitations. For example, the upfront strategy requires a lifelong therapy, which could be impaired the patients' quality of life. Furthermore, in case of TKI-refractory disease, the upfront approach would leave very few treatment options. Then, even though the systemic therapy in DTC is generally effective and well tolerated, the treatment strategy should be planned by a multidisciplinary team, on a case-by-case basis.

In conclusion, the results achieved by lenvatinib in treatment of MDTC are clear and irrefutable. Real-life data, obtained by case reports and retrospective studies, are equally important to increase the knowledge about this drug and improve the clinical management. The optimal approach to thyroid cancer remains multidisciplinary, involving surgeons, radiation therapists, endocrinologists and oncologists, but due to the availability active systemic drugs, such as lenvatinib, current treatment paths should be redesigned.

\section{Summary points}

- Differentiated thyroid carcinoma (DTC) is the most common thyroid cancer.

- Metastatic disease is rare, but not occasional.

- Metastatic disease has few treatment options.

- Lenvatinib showed great efficacy in metastatic DTC.

- A 37-year-old man with DTC was described.

- Lenvatinib therapy was performed.

- The patient was responding to lenvatinib for 22 months.

- The patient noticed a very important quality of life improvement. 
Informed consent

Patient gave written informed consent to describe his case.

\section{Open access}

This work is licensed under the Attribution-NonCommercial-NoDerivatives 4.0 Unported License. To view a copy of this license, visit http://creativecommons.org/licenses/by-nc-nd/4.0/

\section{References}

Papers of special note have been highlighted as: $\bullet$ of interest; $\bullet \bullet$ of considerable interest

1. La Vecchia C, Malvezzi M, Bosetti C et al. Thyroid cancer mortality and incidence: a global overview. Int. J. Cancer 136(9), 2187-2195 (2015).

2. Miccoli P, Sohail B. Surgical management of papillary thyroid carcinoma: an overview. Updates Surg. 69(2), 145-150 (2017).

3. Lamartina L, Durante C, Filetti $S$ et al. Low-risk differentiated thyroid cancer and radioiodine remnant ablation: a systematic review of the literature. J. Clin. Endocrinol. Metab. 100(5), 1748-1761 (2015).

4. Verburg FA, Mäder U, Reiners $\mathrm{C}$ et al. Long-term survival in differentiated thyroid cancer is worse after low-activity initial post-surgical 131I therapy in both high-and low-risk patients. J. Clin. Endocrinol. Metab. 99(12), 4487-4496 (2014).

5. Brose MS, Nutting CM, Jarzab B et al. Sorafenib in radioactive iodine-refractory, locally advanced or metastatic differentiated thyroid cancer: a randomized, double-blind, Phase III trial. Lancet 384(9940), 319-328 (2014).

6. Yamamoto Y, Matsui J, Matsushima T et al. Lenvatinib, an angiogenesis inhibitor targeting VEGFR/FGFR, shows broad antitumor activity in human tumor xenograft models associated with microvessel density and pericyte coverage. Vasc. Cell. 6, 18 (2014).

7. Schlumberger M, Tahara M, Wirth LJ et al. Lenvatinib versus placebo in radioiodinerefractory thyroid cancer. N. Engl. J. Med. 372(7), 621-630 (2015).

- This is the article that every clinician should know, in which the activity of lenvatinib in DTC was demonstrated in a Phase III randomized trial, challenging the clinical practice.

8. Cabanillas ME, Schlumberger M, Jarzab B et al. A Phase II trial of lenvatinib (E7080) in advanced progressive, radio-iodine refractory, differentiated thyroid cancer: a clinical outcome and biomarker assessment. Cancer 121(16), 2749-2756 (2015).

9. Klastersky JA. Adverse events of targeted therapies. Curr. Opin. Oncol. 26(4), 395-402 (2014).

.. This is an interesting article that made the point about the most common target therapies related toxicities and their management in clinical practice. 